\title{
Evidence-based patient choice and psychiatry
}

Evidence-based medicine and patient centred care are natural bedfellows. Muir Gray coined the term evidence-based patient choice, ${ }^{1}$ which provides the title for Elwyn and Edward's edited collection of commissioned papers addressing a wide range of relevant perspectives. ${ }^{2}$ Both evidence-based medicine and patient centred care have developed as a critical response to what is perceived to be the excessive authority given in traditional medicine to physicians and other health professionals. Evidence-based medicine emphasises the point that it is not the authority of the physician that justifies a particular clinical intervention but the evidence for that intervention's effectiveness. Patient centred medicine emphasises the point that patients should play a central role in decisions about their health care. Providing evidence-based knowledge to patients should enhance their power and aid the development of increasingly effective patient centred health care.

Psychiatry is a particularly challenging area of medical practice in which to promote evidence-based patient choice, because mental illness can affect both understanding and decision making abilities. However, the danger is that psychiatrists may too readily assume, in a particular case, that a patient cannot cope with information and choice. As the evidence base in psychiatry increases, so do the opportunities for respecting the autonomy of mentally ill patients.

\section{Patient choice}

There are 3 ethical reasons for the importance of patient choice:

(1) It is a "rights" issue. Patient autonomy ought to be respected for its own sake.

(2) It is instrumental in producing good consequences, ${ }^{34}$ although good evidence supporting this is still lacking. ${ }^{5}$

(3) What counts as a good outcome is, in part, determined by patients' individual values. For example, for one patient, survival for 6 months may be worthwhile; for another patient in an identical clinical state, such survival may seem of little value.

My own view is that a genuine respect for patient choice is good in itself, even if it leads to poorer health.

\section{Evidence-based patient choice}

Evidence-based patient choice brings together evidence-based medicine and patient centred medicine. ${ }^{1}$ Four steps make up what I think of as the evidence-based patient choice chain.

\section{THE ISSUE IS IMPORTANT TO PATIENTS MAKING CHOICES}

The starting point for evidence-based patient choice should be an issue of importance to patients. Patients need to be involved in identifying these issues. This raises the difficult question of who represents patients. Some believe that the best patient representatives are general patient organisations (eg, community health councils) and a selection of actual patients. Patients (or consumers) need to be involved throughout the research process (designing and choosing outcome measures) if patient choice is to be maximised.

\section{THE EVIDENCE MUST BE OF GOOD QUALITY}

The specific contribution of evidence-based medicine to patient choice is the insistence that the evidence be of good quality. The quality of evidence comes in all shades. No clear line of demarcation can be drawn between good quality and poor quality evidence. If the line is drawn too high (eg, allowing only systematic reviews of randomised controlled trials to count as good evidence) then too much information that is likely to interest patients is excluded. To support evidence-based patient choice, the evidence should be based as much as possible on systematic reviews - preferably randomised controlled trials but also systematic reviews of observational studies.

\section{INFORMATION SHOULD BE IN A FORM THAT IS} ACCESSIBLE TO PATIENTS BUT WITH MINIMUM BIAS Much of the information likely to be the focus of evidence-based patient choice is complex in nature. Often it concerns the probabilities of risks and benefits. Care should be taken to ensure that such information is accessible to most patients and that it is presented in a way that does not manipulate patient choices. These points have been discussed above. In order to achieve the aims of both accessibility and lack of manipulation, the information often needs to be presented in various ways.

\section{THE INFORMATION CAN BE USED BY PATIENTS TO ENHANCE CHOICE}

Information by itself is not sufficient to prompt patients to exercise choice. In order to exercise choice, patients need to have the power to choose. Thus, patients must be genuinely involved in the process of making healthcare decisions. This requirement has implications both for the way in which healthcare services are set up and for the way in which patient consultations are done. An important focus for research into patient choice is on ways to enhance patients' abilities to make choices.

Entwistle et al identified 3 criteria for what they call "evidence-informed patient choice": 6

\section{Giving information to patients within the consultation}

Providing patients with high quality information is a key issue in enhancing patient choice. Even patients who do not wish to make final decisions about their health care may still want information. ${ }^{78}$

Different patients have different values, and the best intervention for one patient may not be best for another facing a similar clinical situation. Therefore, 2 methods can be used to factor individual patient values into the decision: either put the patients' values into the decision analysis or give patients the information and allow them to make the choice.

The first method offers a halfway house between oldfashioned paternalism and respect for patient choice. It differs from paternalism because the patient's values are used to arrive at the decision (rather than the physician's own values or the physician's assumption about the patient's values). But the decision process remains with the physician.

The second approach, which is more in keeping with enhancing patient choice, is to provide the information about side effects and treatment benefits to the patient and then let the patient make the choice - in consultation, of course, with the physician as desired. Physicians also have an important role in helping patients understand not only the information but also its quality. 
2 Giving information to patients outside the consultation If patients are to be truly empowered, they will need access to information independently of physicians. Unfortunately, the quality of some information may be very low, which is the case, for example, with many patient leaflets and with information on the internet. Poor quality information does not respect patient autonomy and can lead to patients making the wrong decisions; therefore, the quality of information is an ethical issue. If health information professionals are to play an important role in informing patients - and indeed in informing professionals then they must become skilled at assessing information quality. The DISCERN instrument may help to achieve this. ${ }^{9}$ It provides a method of judging the quality of written consumer health information on treatment choices, and it highlights the issue of whether information is based on high quality evidence-based medicine. DISCERN also emphasises the importance of providing choices and the need for clarity.

\section{Evidence-based medicine and guidelines}

Recommendations and protocols are important outcomes from the application of evidence-based medicine. Treatment protocols are increasingly being devised on the basis of good quality trials of effectiveness. These protocols then set the standard for treatment, and indeed it may be medically negligent not to follow the protocols. Guidelines can benefit patients because they can improve medical practice. However, they can also work to reduce patient choice if patients in a particular clinical situation are simply given the recommended treatment.

Most patients seen in psychiatric practice are able to understand evidence and make choices about their treatment, with support from their physician or other therapist. Many psychological treatments can only be undertaken with the patient's full understanding and acceptance of the treatment method. But mental illness can affect a patient's thinking such that the paradigm of evidence-based patient choice is not appropriate.
At one extreme is the patient with such marked psychosis, depression, or dementia that the physician must take full control of treatment and act in the patient's best interests. The psychiatrist here is in a similar situation to the physician or surgeon faced with a patient in an acute confusional state.

The most challenging situation that psychiatrists face, with regard to evidence-based patient choice, is when the mental illness affects a patient's values but not her intellectual abilities. Examples of such illnesses include mild to moderate depression, mild manic illness, and anorexia nervosa. In such cases patients may make choices based on values that are likely to be substantially different from those that the patient holds when she is well. If the patient's choices have serious consequences, it may be appropriate to enforce treatment through use of the Mental Health Act. In less serious situations, the issue for the psychiatrist may be how far to go in trying to persuade the patient to follow the course of treatment that fits with the values she is likely to have when she is well.

TONY HOPE, MA, PhD, BM BCH Institute of Health Sciences Oxford, UK

1 Hope T. Evidence-based patient choice. London: King's Fund Publishing, 1996.

2 Edwards A, Elwyn G, editors. Evidence-based patient choice: inevitable or impossible? Oxford: Oxford University Press, 2001.

Stewart MA. What is a successful physician-patient interview? A study of interactions and outcomes. Soc Sci Med 1984;19:167-75.

4 Greenfield S, Kaplan SH, Ware JE Jr, et al. Patients' participation in medical care: effects on blood sugar control and quality of life in diabetes.J Gen Intern Med 1988;3:448-57.

5 Toop L. Primary care: core values. Patient centred primary care. BMJ 1998;316:1882-3.

6 Entwistle VA, Sheldon TA, Sowden A, et al. Evidence-informed patient choice. Practical issues of involving patients in decisions about health care choice. Practical issues of involving patients in decisions about

7 Blanchard CG, Labrecque MS, Ruckduschel JC et al. Information and decision-making preferences of hospitalised adult cancer patients. Soc Sci

8 Beisecker AE, Beisecker TD. Patient information-seeking behaviors when communicating with doctors. Med Care 1990;28:19-28.

9 Charnock D. The DISCERN handbook. Oxford: Radcliffe Medical Press, 1998. 